\title{
Aspectos clínicos y microbiológicos de la peritonitis asociada a diálisis peritoneal en pacientes adultos con insuficiencia renal crónica en el Servicio de Urgencias
}

\author{
Pablo San Juan M., Angélica Pérez J. y Cornelio Barrientos A.
}

\section{Clinical and microbiological aspects of peritonitis associated with peritoneal dialysis in adult patients with chronic renal failure in the Emergency Department}

Background: Peritonitis is the most important and frequent complication of peritoneal dialysis (PD). Bacterial infections are responsible in most cases, with characteristic symptoms. Aim: To determine the most frequent pathogens in peritonitis associated with PD in patients with chronic renal failure (CRF). Methodos: Retrospective, descriptive, cross-sectional and observational study of patients with peritonitis in PD with CRF of the emergency department, between July 2012 and June 2013. Sociodemographic, diagnostic, clinical and microbiological and cellular data were evaluated from the patient's fluid. Dialysis. Results: From 73 reviewed records, $52 \%$ were male. The primary cause of CRF was diabetes mellitus type 2 (67\%). Symptoms presented: abdominal pain (86\%), vomiting (42\%) and nausea (34\%), with anemia, azotemia, hyperglycemia, hypoalbuminemia and hyponatremia. The positive microbiological cultures were 59\%; and the pathogens identified were Candida tropicalis $(9.6 \%)$, Staphylococcus epidermidis (8.2\%), Enterococcus faecalis and Staphylococcus haemolyticus (6.8\% each one), associated with elevated leukocytes, azotemia and high cellularity in peritoneal fluid $(\mathrm{p}<0.05)$. Conclusions: The main microorganism determined was of fungal origin associated with leukocytes, azotemia and high cellularity.

Key words: Candida albicans; peritonitis; continuous ambulatory peritoneal dialysis; chronic renal disease; hyponatremia; hypoalbuminemia.

Palabras clave: Candida albicans; peritonitis; diálisis peritoneal; continua ambulatoria; enfermedad renal crónica; hiponatremia; hipoalbuminemia.

\section{Introducción}

a enfermedad renal crónica (ERC) se define como la presencia de una alteración estructural o

funcional renal (sedimento, imagen, histología) que persiste más de tres meses, con o sin deterioro de la función renal o un filtrado glomerular $(\mathrm{FG})<60 \mathrm{ml} /$ $\min / 1,73 \mathrm{~m}^{2}$, sin otros signos de daño renal; es la resultante de diversas enfermedades crónico-degenerativas como: la hipertensión arterial (HTA) sistémica, la diabetes mellitus tipo 2 (DM-2) y las glomerulopatías ${ }^{1,2}$. La terapia de reemplazo renal (TRR) definida como cualquier terapia de depuración extracorpórea que propone sustituir la función renal, ha tenido un crecimiento y genera un desafío económico para los sistemas de salud, en América Latina. La incidencia y prevalencia de la TRR aumenta cada año y no todos los pacientes diagnosticados con enfermedad renal crónico terminal (ERCT) tienen acceso a ella ${ }^{3}$. La diálisis peritoneal (DP) es el tratamiento de primera elección como TRR; existen tres modalidades: continua ambulatoria (DPCA), automatizada (DPA) e intermitente (DPI) ${ }^{4}$.
En México, el método dialítico mayormente empleado es la modalidad peritoneal (80\%); en el mundo representa alrededor de $25 \%$ de toda la población con DP y en la actualidad se considera una pandemia ${ }^{2-4}$.

El sistema de la DP está integrado por cuatro componentes: la sangre capilar, la membrana peritoneal, los vasos linfáticos y el líquido de diálisis. Este sistema terapéutico opera mediante el paso de sustancias de la sangre del paciente al líquido peritoneal y viceversa. A su vez, la membrana peritoneal es una suma de endotelio capilar, intersticio y mesotelio peritoneal ${ }^{5,6}$.

La peritonitis es una de las complicaciones más frecuente de la DP, que impacta en la calidad de vida, supervivencia y estado nutricional del paciente, y se caracteriza por la existencia de líquido del dializado turbio, dolor abdominal y un examen citológico de líquido de diálisis con más de 100 leucocitos $/ \mathrm{mm}^{3}$ con al menos $50 \%$ de polimorfonucleares ${ }^{7}$, lo que podría responder a una irritación química, necrosis local, contusión directa o invasión bacteriana, siendo la causa más frecuente del fracaso terapéutico de una $\mathrm{DP}^{8}$. Se clasifican en primarias (peritonitis monomicrobianas, en las que no se evidencia
Instituto Mexicano del Seguro Social. México, D.F., México. Hospital General Regional № 6 (PSJM).

Instituto Politécnico Nacional Escuela Superior de Medicina (APJ, CBA).

Los autores declaran no tener conflicto de intereses.

Trabajo fue financiado con fondos del IMSS, Servicio de Urgencias del $\mathrm{HGZ} \mathrm{N}^{\circ} 6$

Recibido: 11 de septiembre de 2017

Aceptado: 8 de marzo de 2018

\section{Correspondencia a:}

Cornelio Barrientos Alvarado cornelio_barrientos@yahoo. com.mx 
un foco infeccioso intra-abdominal), secundarias (peritonitis polimicrobianas con origen intra-abdominal) y terciarias (peritonitis persistentes y recurrentes), que son complicadas 9 . La peritonitis bacteriana es la principal complicación de la DPCA, y para los pacientes con insuficiencia renal crónica (IRC) es la principal causa de morbilidad y mortalidad, ocasionando el mayor porcentaje de retiro del catéter ${ }^{10}$. Existen diversos mecanismos causantes de peritonitis; entre los más comunes se encuentran: contaminación del catéter, infección del sitio de salida o del túnel cutáneo del catéter, translocación bacteriana gastrointestinal y bacteriemia ${ }^{11}$.

Los microorganismos grampositivos son los agentes causales más frecuentes de peritonitis asociada a diálisis, contribuyendo en 60 a $80 \%$ de éstos. Staphylococcus epidermidis, Staphylococcus aureus y Streptococcus spp se pueden encontrar en 27 a 45,10 a 20 y 5 a $10 \%$ de los casos, respectivamente. Otras especies grampositivas son menos frecuentes, excepto Enterococcus spp en pacientes pediátricos, donde alcanza hasta $37,7 \%$ en algunas series. Por otra parte, bacterias gramnegativas como Pseudomonas aeruginosa, Escherichia coli y Klebsiella pneumoniae, son causantes de peritonitis en 7,$1 ; 6,8$ y $5,2 \%$, respectivamente. La peritonitis fúngica es infrecuente pero no ausente, especialmente en pacientes que han recibido múltiples cursos de antimicrobianos ${ }^{12}$.

Es recomendable siempre cultivar el líquido peritoneal para establecer la etiología del episodio y estudiar la resistencia a antimicrobianos para eventuales ajustes terapéuticos al esquema antibacteriano empírico inicial ${ }^{13}$. El conocer la etiología infecciosa de la peritonitis, en pacientes tratados con DPCA, es de gran importancia para un mejor manejo del paciente y minimizar las implicaciones económicas que conlleva el tratamiento, tanto para el paciente como para la institución de salud ${ }^{14}$.

El objetivo de este estudio fue describir los hallazgos clínicos y microbiológicos en peritonitis asociados con la DP en pacientes con IRC que ingresan en el servicio de urgencias, determinando los factores de riesgo y el tiempo de la peritonitis en el programa de DPCA.

\section{Pacientes y Métodos}

Para la realización del presente estudio, de 547 registros de pacientes con IRC, se revisaron 73 expedientes de pacientes con peritonitis en DP al ingreso al Servicio de Urgencias del Hospital General Regional $\mathrm{N}^{\circ} 6$ de Cd. Madero Tamaulipas, en el período comprendido entre junio de 2012 y julio de 2013.

\section{Criterios de inclusión}

Pacientes con IRC en estadio 5 de K-DOQI en tratamiento sustitutivo mediante DP, con primer episodio de peritonitis y cultivo de líquido de diálisis en el Servicio de Urgencias.

\section{Criterios de exclusión}

Pacientes con insuficiencia hepática Child-Pugh B o C, infección por $\mathrm{VIH}$, pacientes en tratamiento antimicrobiano para peritonitis y pacientes con tunelitis (infección en el sitio de inserción del catéter). Se eliminaron del estudio aquellos pacientes cuyo expediente estuviera incompleto.

Se realizó un estudio observacional, transversal, descriptivo y retrospectivo en pacientes con IRC y diagnóstico de peritonitis asociada con DP, utilizando el método de revisión de registros clínicos y nota de egreso del Servicio de Urgencias.

Se consignó en una planilla de excel los datos sociodemográficos, las causas de la IRC, el tiempo de evolución de las co-morbilidades asociadas, hallazgos relevantes de laboratorio: biometría hemática y química sanguínea, el recuento celular y estudio microbiológico del líquido de diálisis, y la historia renal donde se desglosa el tiempo de inicio de la diálisis, adiestramiento, número de recambios, tipo de líquido de diálisis.

Debido a que fue un estudio con riesgo menor al mínimo, no se requirió firma del consentimiento informado, siendo la unidad de estudio el expediente clínico, dispensado por el Comité de Ética de Investigación del hospital, quien aprobó el protocolo.

\section{Análisis estadístico}

Una vez recopilados los datos se procedió a la codificación de las variables. Después se elaboró una base de datos en el programa IBM SPSS Statistics versión 22. El análisis estadístico descriptivo se realizó con medidas de tendencia central y de dispersión para las variables cuantitativas. La distribución teórica normal de las variables cuantitativas fue evaluada con la prueba de Kolmogorv-Smirnov, mientras que las variables cualitativas se describieron con distribución de frecuencias y porcentaje. Por la prueba de $t$ de muestras independientes se evaluó las diferencias de cultivos positivos con respecto a los negativos de los valores de: hemoglobina, hematocrito, leucocitos, neutrófilos, glucosa, nitrógeno ureico en la sangre, creatinina, potasio, sodio, albumina y celularidad. Y por la prueba de $\chi^{2}$ se determinó la asociación de cultivos positivos y negativos entre adiestramiento, número de recambios y episodios previos. La probabilidad $\alpha$ es de 0,05 .

\section{Resultados}

De acuerdo al censo de pacientes con IRC en la unidad de diálisis de la institución, 547 pacientes fueron registraron durante el período de estudio del 01 de julio de 2012 al 30 de junio de 2013. Setenta y tres expedientes de estos 
pacientes: 13,3\% (73/547), que habían ingresado por el Servicio de Urgencias con el diagnóstico de peritonitis, se incluyeron en este estudio. La DPCA se registró en $100 \%$ en este estudio.

La edad media fue de 61,5 $\pm 0,8$ años, siendo del género masculino $50,6 \%$.

Como causas primarias de la IRC en el programa de diálisis, la DM-2 representó 67,1\%. Las principales comorbilidades que presentaban los pacientes del grupo de estudio fueron: la HTA sistémica $(84,3 \%)$ y DM-2 $(79,4 \%)$ (Tabla 1).

Más de $50 \%$ de los pacientes presentaron peritonitis en los primeros meses del programa de DPCA, 45,2\% (33 pacientes) dentro de los primeros cinco meses posteriores a la colocación del catéter, 24,6\% (18 pacientes) dentro de los 6 a 12 meses, y sólo 1,4\% (un paciente) a más de 49 meses (Figura 1).

Los síntomas encontrados y por el cual acudieron al Servicio de Urgencias fueron: dolor abdominal difuso $86,3 \%$ (63 pacientes), vómitos $46,6 \%$ (34 pacientes) y náuseas $41,1 \%$ (30 pacientes). Sólo $19,2 \%$ (14 pacientes) presentaron fiebre al ingreso (Figura 2).

Al ingreso en el servicio de urgencias se les realizó evaluación de laboratorio encontrando los siguientes índices propios de la peritonitis: un nivel promedio de hemoglobina y hematocrito menores a los rangos normales: $10,29 \mathrm{~g} / \mathrm{dL}$ y $28,6 \%$, respectivamente, recuento de leucocitos de $10.800 / \mathrm{mm}^{3}$ con 8.270 neutrófilos/ $\mathrm{mm}^{3}$. Glicemia elevada e hipoalbuminemia, valor normal de potasemia e hiponatremia leve con un promedio de $134,23 \mathrm{mmol} / \mathrm{L}$. Tomando en cuenta que los pacientes se encontraban en un programa de DP, se registraron valores
Tabla 1. Características socio-epidemiológicas de pacientes con peritonitis asociada a diálisis peritoneal continua ambulatoria $(n=73)$

\begin{tabular}{|c|c|c|c|c|}
\hline Variables & & $\overline{\mathrm{x}}$ & (DE) & Mín-Máx \\
\hline \multirow[t]{2}{*}{ Edad } & & 61,5 & $(7,45)$ & $47-81$ \\
\hline & & \multicolumn{2}{|c|}{ n (pacientes) } & Total \\
\hline \multirow[t]{2}{*}{ Sexo } & Masculino & & 37 & \multirow[t]{2}{*}{73} \\
\hline & Femenino & & 36 & \\
\hline \multirow{2}{*}{$\begin{array}{l}\text { Causa primaria de la } \\
\text { insuficiencia renal crónica }\end{array}$} & Diabetes mellitus tipo 2 & & 49 & \multirow[t]{2}{*}{73} \\
\hline & Hipertensión arterial sistémica & & 24 & \\
\hline \multirow[t]{8}{*}{ Co-morbilidades } & Hipertensión arterial sistémica & & 62 & \multirow[t]{8}{*}{73} \\
\hline & Diabetes mellitus tipo 2 & & 58 & \\
\hline & Cardiopatía isquémica & & 30 & \\
\hline & Enfermedades vascular periférica & & 19 & \\
\hline & Insuficiencia cardiaca congestiva & & 13 & \\
\hline & Enfermedades gastrointestinales & & 9 & \\
\hline & Evento vascular cerebral & & 4 & \\
\hline & EPOC & & 1 & \\
\hline
\end{tabular}

EPOC: enfermedad pulmonar obstructiva crónica.

superiores dentro de los rangos normales de creatininemia y nitrógeno ureico. El líquido peritoneal se describió con turbidez en $100 \%$ de los casos y se reportó una media general de 1.745 céls $/ \mathrm{mm}^{3}$ (Tabla 2).

El cultivo de líquido de diálisis demostró el desarrollo de: Candida tropicalis $(9,6 \%)$, Staphylococcus epidermidis (8,2\%), Enterococcus faecalis y Staphylococcus haemolyticus (6,8\% cada uno) y Candida parapsilosis (5,5\%). En 41,1\% de los pacientes no hubo desarrollo microbiano (Tabla 3).

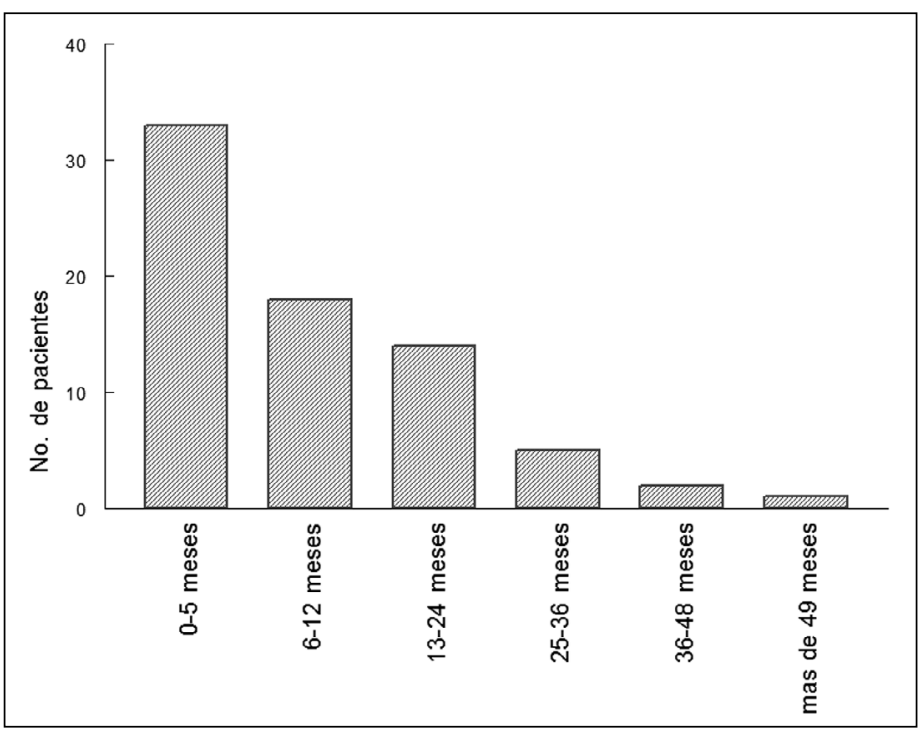

Figura 1. Lapso de tiempo estimado entre el inicio de la diálisis peritoneal y la detección de peritonitis asociada a diálisis peritoneal continua ambulatoria $(n=73)$.

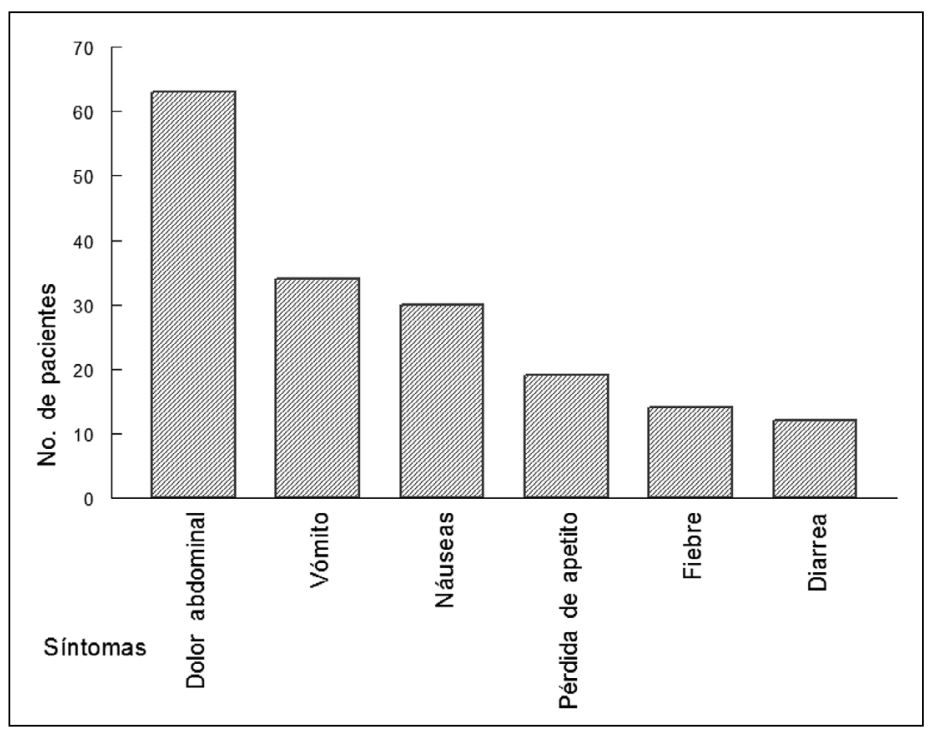

Figura 2. Manifestaciones clínicas de peritonitis asociada a diálisis peritoneal continua ambulatoria $(n=73)$. 
Tabla 2. Parámetros de laboratorio promedio en pacientes con peritonitis asociada a diálisis peritoneal continua ambulatoria $(n=73)$

\begin{tabular}{llcc}
\hline Variables paraclínicas & & $\overline{\mathrm{x}}$ & (DE) \\
Hemograma & Hemoglobina $(\mathrm{g} / \mathrm{dL})$ & 10,29 & 1,08 \\
& Hematocrito $(\%)$ & 28,6 & 0,49 \\
& Leucocitos $/ \mathrm{mm}^{3}$ & 10.800 & 794 \\
& Neutrófilos $/ \mathrm{mm}^{3}$ & 8.270 & 663 \\
\hline Bioquímica sanguínea & Glucosa $(\mathrm{mg} / \mathrm{dL})$ & 150,76 & 7,85 \\
& Albumina $(\mathrm{g} / \mathrm{dL})$ & 2,73 & 0,08 \\
& Potasio $(\mathrm{mmol} / \mathrm{L})$ & 4,05 & 0,10 \\
& Sodio $(\mathrm{mmol} / \mathrm{L})$ & 134 & 0,58 \\
\hline Pruebas de función renal & Creatinina $(\mathrm{mg} / \mathrm{dL})$ & 6,93 & 0,34 \\
& N ureico $(\mathrm{mg} / \mathrm{dL})$ & 55,89 & 3,34 \\
\hline Líquido de diálisis & Células & 1.745 & 427 \\
\hline Se expresa la media \pm el ES. & & & \\
\hline
\end{tabular}

Al comparar los parámetros de laboratorio entre un origen infeccioso de las peritonitis y un presunto no infeccioso, se observó que el recuento de leucocitos, la creatinina y la celularidad en el líquido de diálisis, fueron significativamente mayores en la peritonitis demostradamente infecciosa (Tabla 4).

Al hacer la asociación entre la peritonitis infecciosa y presunta no infecciosa con respecto a los parámetros de historia renal, se observó una dependencia significativa con respeto al adiestramiento $(\mathrm{p}=0,04)$, número de recambios $(\mathrm{p}=0,001)$ y episodios previos de diálisis $(\mathrm{p}=0,001)$ (Figura 3).
Tabla 3. Microorganismos recuperados del líquido de diálisis en pacientes con insuficiencia renal crónica y peritonitis asociada a diálisis peritoneal continua ambulatoria $(n=73)$

$\left.\begin{array}{lrl}\hline \text { Microorganismos } & 30 & (41,1) \\ \hline \text { Sin desarrollo de microorganismos } & 7 & (9,6) \\ \hline \text { Candida tropicalis } & 6 & (8,2) \\ \hline \text { ftaphylococcuencia }(\%)\end{array}\right)$

Tabla 4. Parámetros de laboratorio promedio en pacientes con peritonitis asociada a diálisis peritoneal continua ambulatoria, con y sin infección, ( $\mathrm{n}=\mathbf{7 3}$ )

\begin{tabular}{|c|c|c|c|c|c|c|}
\hline \multirow[b]{3}{*}{ Variables paraclínicas } & & \multicolumn{4}{|c|}{ Diagnóstico bacteriológico } & \multirow[b]{3}{*}{$\mathbf{p}$} \\
\hline & & \multirow{2}{*}{$\begin{array}{c}\text { Positivo } \\
\bar{x}\end{array}$} & \multicolumn{3}{|c|}{ Negativo } & \\
\hline & & & (ES) & $\bar{x}$ & (ES) & \\
\hline \multirow[t]{4}{*}{ Hemograma } & Hemoglobina (g/dL) & 11,05 & 1,82 & 9,20 & 0,26 & 0,403 \\
\hline & Hematocrito (\%) & 28,62 & 4,52 & 28,58 & 3,86 & 0,973 \\
\hline & Leucocitos $/ \mathrm{mm}^{3}$ & 12,21 & 1,18 & 8,77 & 0,79 & 0,032 \\
\hline & Neutrófilos/mm³ & 9,01 & 0,98 & 7,19 & 0,75 & 0,179 \\
\hline \multirow[t]{6}{*}{ Bioquímica sanguínea } & Glucosa (mg/dL) & 152,72 & 10,54 & 147,96 & 11,88 & 0,768 \\
\hline & Albumina (g/dL) & 2,65 & 0,10 & 2,85 & 0,14 & 0,252 \\
\hline & Potasio (mmol/L) & 4,09 & 0,14 & 4,01 & 0,13 & 0,707 \\
\hline & Sodio (mmol/L) & 133 & 0,77 & 135,50 & 0,83 & 0,067 \\
\hline & Creatinina $(\mathrm{mg} / \mathrm{dL})$ & 7,78 & 0,45 & 5,72 & 0,40 & 0,002 \\
\hline & $\mathrm{N}$ ureico $(\mathrm{mg} / \mathrm{dL})$ & 58,37 & 3,45 & 52,33 & 6,46 & 0,377 \\
\hline Líquido de diálisis & Células & $2.676,86$ & 681,16 & 409,33 & 184,86 & 0,008 \\
\hline
\end{tabular}

Se expresa la media \pm el ES. Prueba t de student, $\mathrm{p}<0,05$. 


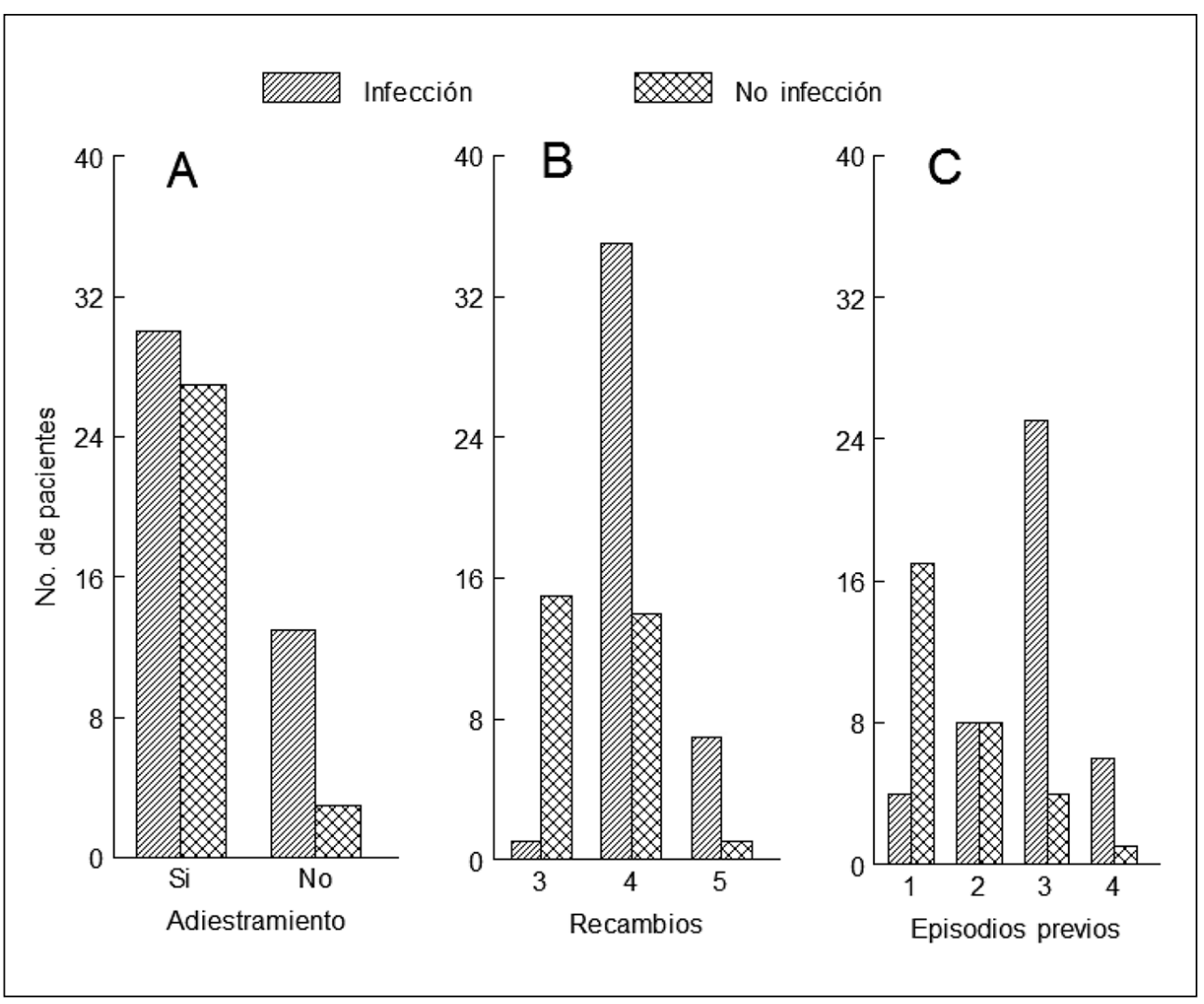

Figura 3. Frecuencia de peritonitis infecciosa y no infecciosa asociado a diálisis peritoneal continua ambulatoria: (A) con y sin adiestramiento, (B) número de recambios y (C) episodios previos, $(n=73)$. Chi cuadrada. $p<0,05$.

\section{Discusión}

La ERC es resultante de diversas enfermedades crónico degenerativas, entre las que destacan la DM-2 e HTA. En México hay alta prevalencia de enfermedades crónico degenerativas como la DM-2 y la HTA, que han desplazado a las enfermedades infecciosas entre las principales causas de muerte ${ }^{15}$. No obstante, éstas pueden ser causa primaria para el desarrollo de infecciones; en este estudio se observó que tanto la DM-2 como la HTA, son los principales factores encontrados en los pacientes con IRC (Tabla 1).

$\mathrm{Y}$ aunque se reporta que la incidencia de la peritonitis asociada a diálisis está relacionada al tipo de patología condicionante de la IRC, también está relacionada a otros factores como: edad, el género e inicio de la terapia dialítica y a las posibilidades de que los pacientes tengan acceso a cuidados de salud ${ }^{16-18}$.

La edad promedio en este estudio (Tabla 1) osciló alrededor de los 61 años; este podría ser un factor de riesgo pues se ha reportado la edad avanzada como un rasgo destacado en los pacientes con peritonitis ${ }^{19}$.

El predominio de varones observado puede ser debido a la mayor población de pacientes masculinos inscritos en el programa de DP en la unidad donde se llevara a cabo el estudio, puesto que algunos autores señalan una mayor incidencia en el sexo femenino ${ }^{20}$ y otros en el masculino ${ }^{19}$.

En México no existe un registro nacional de pacientes con ERC que nos permita conocer con precisión las co-morbilidades asociadas ${ }^{21}$. La DM-2 y luego la HTA se reportan como las principales causas de daño renal crónico $^{22,23}$ (Tabla 1), siendo su prevalencia similar a la de países desarrollados ${ }^{3}$, su magnitud reviste caracteres de epidemia ${ }^{24}$.

Otros factores de riesgo reportado en la peritonitis son el tiempo prolongado de permanencia en programa de DP y el tiempo desde la inserción del catéter peritonea ${ }^{25,26}$. En este estudio llama la atención que en el primer año cerca de $70 \%$ presentaron peritonitis, sin embargo, predominaron más durante los primeros cinco meses (Figura 1), hecho probablemente asociado a la técnica quirúrgica o técnica de diálisis de los pacientes.

Dados estos resultados, se puede sugerir que en la población hace falta centrar la atención en la prevención y el manejo de dichos pacientes.

El mantenimiento del catéter una vez detectada la infección se ha relacionado con un peor pronóstico y constituye en ocasiones el principal factor desencade- 
nante del fallo de la técnica y de la mortalidad ${ }^{18,19,26}$. En las pautas más recientes de la International Society for Peritoneal Dialysis el comité hizo mayor énfasis en la sección sobre prevención, ya que se ha observado que prevenir la peritonitis es una de las claves del éxito de la $\mathrm{DP}^{27}$.

Los hallazgos clínicos en este estudio mostraron que la mayoría de los pacientes con peritonitis presentaron dolor abdominal, vómitos, náuseas y solamente 19,1\% presentaban fiebre (Figura 2), síntomas que coinciden con los informados en otros reportes ${ }^{23,28}$. Esto se debe probablemente a la falta de respuesta inflamatoria, fenómeno secundario a la desnutrición y la supresión inmunológica del paciente. En definitiva, el diagnóstico se realiza por análisis bioquímico del líquido peritoneal cuando se detecta un recuento $\geq 100$ leucocitos $/ \mathrm{mm}^{3}$ (y al menos $50 \%$ de polimorfonucleares), pero el análisis microbiológico es imprescindible para establecer la etiología ${ }^{10,21}$.

Se ha mencionado y se puede sospechar también un episodio de peritonitis cuando acontecen episodios recurrentes, y entre los datos analíticos asociados con peritonitis infecciosa se han descrito como significativo la presencia de anemia ${ }^{29-31}$; en este estudio se encontró que los pacientes presentaban concentraciones inferiores a los normales de hemoglobina y hematocrito (Tabla 2). El recuento sanguíneo de leucocitos y neutrófilos no muestran grandes modificaciones ante la peritonitis en los pacientes con nefropatías; nuestra experiencia (Tabla 2) concuerda con otros estudios, pudiendo concluirse que el recuento leucocitario carece de utilidad como parte de la valoración y diagnóstico de peritonitis en estos pacientes ${ }^{32}$.

La IRC implica la retención de diversos productos de desechos del metabolismo como la urea, nitrógeno ureico y creatinina, estado denominado azoemia ${ }^{33,34}$.

Se describe que la hiperglicemia es un factor de riesgo asociado a peritonitis en $\mathrm{DP}^{35}$; la hiperglicemia es un potente quimio-activador de neutrófilos jugando un papel en la inflamación ya que induce un aumento en el NF-kB (Factor nuclear kappa B) lo cual facilita la generación de infecciones ${ }^{36}$.

La hipoalbuminemia propia de pacientes con IRC conlleva un descenso en diversas proteínas, muchas de las cuales pueden ser necesarias para el adecuado funcionamiento del sistema inmunitario (p. ej. citoquinas $)^{37,38}$. La hipoalbuminemia que se presentó en este estudio (Tabla 2) podría explicarse por el déficit de ultrafiltración que acontece durante el episodio de peritonitis o por el incremento de la pérdida peritoneal de proteínas, factor que se ha relacionado con un peor pronóstico ${ }^{39}$. Huerta y cols, en el 2010, realizaron un estudio donde evaluaron los niveles de albumina en paciente con peritonitis y determinaron que la hipoalbuminemia se asocia con una elevación hasta 16 veces del riego de peritonitis en pacientes en $\mathrm{DPCA}^{40}$.
Dentro de los criterios diagnósticos de peritonitis asociada a diálisis se presenta un recuento celular elevado ( $>100$ céls $/ \mathrm{mm}^{3}$ ) con predominio de neutrófilos, leucocitos y demostración de bacterias ${ }^{41}$, situación presentada en este estudio en el cual la celularidad fue elevada (Tabla 2), mostrándose una asociación dependiente significativa entre la peritonitis infecciosa y mayor celularidad del líquido peritoneal (Tabla 4).

Al evaluar los resultados de cultivos del líquido peritoneal se observó que menos de la mitad de los cultivos fueron negativos (Tabla 3). Un estudio reportado por Iraj y cols., en el año 2011 describió una prevalencia de cultivos negativos de $55,9 \% \%^{42}$, esto es importante ya que manejo de la peritonitis con cultivo negativo es desafiante por la incertidumbre del diagnóstico y por la falta de evidencia para tomar una decisión terapéutica. Sin embargo, la peritonitis infecciosa en pacientes en tratamiento con diálisis peritoneal está originada comúnmente por bacterias, en algunos casos la etiología es mixta (bacteriana y fúngica) o polimicrobiana ${ }^{43-45}$. Para este estudio, los resultados obtenidos en los cultivos del líquido de diálisis positivos, comprobaron que el principal microorganismo aislado fue C. tropicalis, seguido de $S$. epidermidis. Sin embargo, llama la atención la presencia de $C$. parapsilosis $(5,5 \%)$ y C. albicans $(4,1 \%)$. La peritonitis fúngica aparece generalmente tras terapias reiteradas de antibacterianos de amplio espectro o en enfermos debilitados, y en las perforaciones intestinales. Es un cuadro grave y la retirada del catéter debe realizarse lo antes posible, porque cuanto más se demore, mayor es el riesgo de muerte ${ }^{46}$, se trata de una complicación infrecuente y su incidencia es similar en la DPA y la DPCA, aunque el menor número de conexiones puede reducir los episodios en la técnica automatizada. Aunque la infección fúngica tiene una frecuencia de alrededor de 1 a $23 \%$ en adultos ${ }^{47}$, factor primordial de riesgo para su desarrollo son los episodios previos de peritonitis bacteriana ${ }^{48}$.

Es importante enfatizar que el espectro de microorganismos causantes de peritonitis ha cambiado; los episodios por Staphylococcus coagulasa negativa se han reducido, aumentando proporcionalmente los causados por bacilos gramnegativos. Se está demostrando, también en nuestra casuística lo comprobamos, que la peritonitis fúngica ha ido aumentando su frecuencia y aunque la infección fúngica es infrecuente, se asocia con una alta morbilidad, con la imposibilidad de continuar en el programa de diálisis y con un importante índice de mortalidad ${ }^{49}$.

Las defensas peritoneales tienen una acción protectora de la cavidad peritoneal frente a la invasión de un microorganismo, relacionada con la actividad fagocítica de los leucocitos y con los factores inmunológicos; la inmunidad local tiene un importante papel en la prevención y resolución de la peritonitis ${ }^{50}$, en este estudio se mostró 
una elevación significativa de los niveles de leucocitos en la peritonitis infecciosa (Tabla 4).

Las peritonitis infecciosas, se asociaron, en nuestra casuística, también a factores de adiestramiento, recambio y episodios previos de diálisis peritoneal (Figura 3).

Se concluye que el conocimiento de los factores de riesgo para peritonitis en diálisis peritoneal deriva de estudios multivariados, con control de variables socio-demográficas, causa de la nefropatía, modalidad de DP, etc.

Agradecimientos. Al Instituto Mexicano del Seguro Social, Servicio de Urgencias del HGZ N ${ }^{\circ} 6$.

\section{Resumen}

Introducción: La peritonitis es la complicación más importante y frecuente de la diálisis peritoneal (DP), las infecciones bacterianas son las responsables en la mayoría de los casos, con sintomatología característica. Objetivo: Determinar los patógenos más frecuentes en peritonitis asociado con la DP en pacientes con insuficiencia renal crónica (IRC). Metodología: Estudio retrospectivo, descriptivo, transversal y observacional, de pacientes con peritonitis en DP con IRC del servicio de urgencias, entre julio de 2012 y junio de 2013. Se evaluaron de los expedientes datos sociodemográficos, de diagnóstico, clínicos y microbiológicos y celulares de líquido de diálisis. Resultados: De 73 expedientes revisados 52\% correspondían a pacientes del género masculino, la causa primaria de la IRC fue la diabetes mellitus tipo 2 (67\%). Síntomas presentados: dolor abdominal (86\%), vómitos (42\%) y náuseas (34\%), con anemia, azoemia, hiperglicemia hipoalbuminemia e hiponatremia. Los cultivos microbiológicos positivos fueron 59\%; y los patógenos identificados fueron Candida tropicalis (9,6\%), Staphylococcus epidermidis $(8,2 \%)$, y Enterococcus faecalis y Staphylococcus haemolyticus (6,8\% cada uno), asociados con elevación de leucocitos, azoemia y celularidad alta en el líquido peritoneal $(\mathrm{p}<0,05)$. Conclusiones: El principal microorganismo determinado fue de origen fúngico asociado a leucocitos, azoemia y celularidad alta.

\section{Referencias bibliográficas}

1.- National Kidney Foundation K/DOQI Clinical practice guidelines for chronic kidney disease: evaluation, classification and stratification. Kidney disease outcome quality initiative. Am J Kidney Dis 2002; 39 (2 Supply 1): S1-266. https://www.ncbi.nlm.nih.gov/ pubmed/11904577

2.- Ávila S M N. Enfermedad renal crónica: prevención y detección temprana en el primer nivel de atención. Med Int Mex 2013; 29 : 148-53 http://www.medigraphic.com/pdfs/ medintmex/mim-2013/mim132e.pdf

3.- López M, Rojas M, Tirado L, Durán L, Pacheco R, Venado A, et al. Capítulo 1 Situación actual de la enfermedad renal crónica en México. López M, Rojas M, Tirado L, Durán L, Pacheco R, Venado A, et al. Enfermedad Renal Crónica y su Atención Mediante Tratamiento Sustitutivo en México. 1 ${ }^{\text {a }}$. Edición. México, D.F. Facultad de Medicina, Universidad Nacional Autónoma de México. 2009. P. 19-38.

4.- Su H L, Abascal M A, Méndez B F J, Paniagua R, Amato D. Epidemiologic and demographic aspects of peritoneal dialysis in Mexico. Perit Dial Int 1996; 16 (4): 362-5.

5.- Bernuy J, Cieza J. Tipos de membrana peritoneal y su sobrevida en función al test de equilibrio peritoneal en pacientes en DPCA. Nephrol Dial Transplant. 2004; 19: 2101-6. http://www.upch.edu.pe/vrinve/dugic/revistas/ index.php/RMH/article/view/1140/1167

6.- Selgas R, Bajo M A, Del Peso G. Preserving the peritoneal dialysis membrane in long-term peritoneal dialysis patients. Sem Dial 1995; 8 : 326-32.

7.- $\quad$ Chow K M, Szeto C C, Cheung K K, Leung C B, Wong S S, Law M C, et al. Predictive value of dialysate cell counts in peritonitis complicating peritoneal dialysis. Clin J Am Soc Nephrol 2006; 1: 768-73. DOI: 10.2215/ CJN.01010306

8.- Stuart T, Booth C, Charlotte J, Cash C. Ayshea H, Antony G, et al. Complications of Continuous Ambulatory Peritoneal Dialysis. Radio Graphics 2009; 29: 441-60. 10.1148/ rg.292085136.

9.- Goldstein M, Carrillo M, Ghai S. Continuous ambulatory peritoneal dialysis a guide to imaging appearances and complications. Insights Imaging. 2013; 4: 85-92. doi: 10.1007/ s13244-012-0203-y.

10.- Paredes J C, Rivera C, Durán E, Balladares L. Estudio bacteriológico del paciente con peritonitis debida a diálisis peritoneal continua ambulatoria en el Hospital General de México. Med Int Mex 2006; 22: 172-82 http://www. medigraphic.com/pdfs/medintmex/mim-2006/ mim063c.pdf.

11.- Kavanagh D, Gordon J, Prescott R. Peritoneal dialysis-associated peritonitis in Scotland (1999-2002). Nephrol Dial Transplant 2004; 19: 2584-91. DOI: 10.1093/ndt/gfh386.

12.- Rodríguez C. Diálisis peritoneal automática, Nefrología. 2000; 20 (Supl 2): 46-52.

13.- Ranyel F S. $1^{\text {er }}$ consenso nacional sobre uso de antibióticos en peritonitis secundaria asociada a diálisis peritoneal continua ambulatoria (DPCA). Enf Infec Microbiol 2005; 25 (3): 1-3. http://www.medigraphic.com/pdfs/medintmex/ mim-2005/mim056i.pdf.

14.- Orantes C M, Herrera R, Almaguer M, Brizuela E G, Hernández C E, Bayarre H, et al. Chronic kidney disease and associated risk factors in the Bajo Lempa region of El Salvador: Nefrolempa study, 2009. MEDICC Rev 2011; 13(4): 14-22.

15.- Tamayo O J A. La cobertura nacional de la enfermedad renal crónica. Gac Méd Méx. 2008; 520-3. https://www.anmm.org.mx/GMM/2008/ n6/55_vol_144_n6.pdf.

16.- Korevaar J, Van Manen J G, Boeschotem E V, Dekker F W, Krediet R T. Evaluation of guidelines for peritoneal dialysis patients: A review from the Netherlands Cooperative Study on the adequacy of Dialysis (NECOSAS). Contrib Nephrol 2003; 140: 142-50.

17.- Fried L, Abidi S, Bernardini J, Johnston J R, Priano B. Hospitalization in peritoneal dialysis patients. Am J Kidney Dis 1999; 33: 927-33.

18.- Piraino B, Bailie G R, Bernardini J, Boeschoten E, Amit G, et al. Recomendaciones para las infecciones relacionadas con diálisis peritoneal de la ISPD. Peritoneal Dialysis In. 2005; 25 : 107-10. https://ispd.org/media/pdf/Copy\%20 of\%20ISPD_Guidelines_2005_2_.pdf.

19.- Indhumathi $\bar{E}$, Chandrasekaran $\bar{V}$, Jagadeswaran D, Varadarajan M, Abraham G, Soundararajan P. The risk factors and outcome of fungal peritonitis in continuous ambulatory peritoneal dialysis patients. Indian J Med Microbiol. 2009; 27: 59-61. https://www.ncbi.nlm.nih.gov/ pubmed/19172063.

20.- Wong P N, Lo K Y, Tong G M, Chan S F, Lo M W, Mak S K, et al. Treatment of fungal 
peritonitis with a combination of intravenous amphotericin B and oral flucytosine, and delayed catheter replacement in continuous ambulatory peritoneal dialysis. Perit Dial Int. 2008; 28: 155-62. https://www.ncbi.nlm.nih. gov/pubmed/18332451.

21.- Hernández L, Abascal A, Méndez F J, Paniagua R, Amato D. Epidemiologic and demographic aspects of peritoneal dialysis in Mexico. Perit Dial Int.1996;16: 362-5.

22.- Cruz C, Montenegro J, Olivares M J. Diálisis peritoneal, insuficiencia renal crónica, México, Trillas. 2000; 12: 12-20.

23.- Fried L, Bernardini J, Johnston JR, Priano B. Peritonitis indices mortality in peritoneal dialysis patients. J Am Soc Nephrol 1996; 7: 2176-82.

24.- Paniagua R, Ramos A, Fabián R, Lagunas J, Amato D. Chronic kidney disease and dialysis in Mexico. Perit Dial Int. 2007; 27(4): 405-9. https://www.ncbi.nlm.nih.gov/ pubmed/17602147.

25.- Troidle L, Gorban-Brennan N, Kliger A, Finkelstein FO. Continuous peritoneal dialysisassociated peritonitis: a review and current concepts. Semin Dial 2003; 6: 428-37. https:// www.ncbi.nlm.nih.gov/pubmed/14629601.

26.- Felgueiras J, Del Peso G, Bajo A, Hevia C, Romero S, Celadilla O, et al. Risk of technique failure and death in fungal peritonitis is determined mainly by duration on peritoneal dialysis: single-center experience of 24 years. Adv Perit Dial. 2006; 22: 77-81. http:// europepmc.org/abstract/med/16983945.

27.- Stuart T. Booth C, Charlotte J, Cash C. Ayshea H, Antony G, et al. Complications of Continuous Ambulatory Peritoneal Dialysis. Radio Graphics. 2009; 29: 441-60. 10.1148/ rg.292085136.

28.- Laroche M, Harding G. Primary and secondary peritonitis: an update. Eur J Clin Microbiol Infect Dis 1998; 17: 542-50.

29.- Molina P, Puchades M J, Aparicio M, García Ramón R, Miguel A. Experiencia en peritonitis fúngica en una unidad de diálisis durante diez años. Nefrología 2005; 25: 393-8.

30.- Kocak Z, Bulut C, Kinikli S, Yilmaz G R, Irmak H, Demdroz A P. Fungal peritonitis in patients undergoing continuous ambulatory peritoneal dialysis: A report of three cases. Turk Med J 2007; 1: 30-3. doi: 10.3265/ Nefrologia.2009.29.6.5647.

31.- Ampel N M, White J D, Varanasi U R, Larwood T R, Van Wyck D B, Galgiani J N. Coccidioidal peritonitis associated with continuous ambulatory peritoneal dialysis. Am J Kidney Dis 1988; 11: 512-4.

32.- Li P K, Szeto C C, Piraino B, Bernardini J, Figueiredo A E, Gupta A, et al. Peritoneal dialysis-related infections recommendations: 2010 update. Perit Dial Int 2010; 30: 393-423. doi: 10.3747 pdi.2010.00049.

33.- Brosnahan G, Fraer M. Chronic kidney disease: whom to screen and how to treat, part 1: definition, epidemiology, and laboratory testing. South Med J. 2010; 103(2):140-6. doi: 10.1097/SMJ.0b013e3181c99438.

34.- Eknoyan G.The Renal Section, Department of Medicine, Baylor College of Medicine, Houston, Texas, USA. Chronic kidney disease definition and classification: no need for a rush to judgment. Kidney Int. 2009; 75(10): 1015-8. doi: 10.1038/ki.2009.53

35.- Aguilar M D, Sumarriva P D. Factores de riesgo asociados a infecciones en diálisis peritoneal (DPCA). Rev Esp Med-Quir 2006; 11 (3): 21-4. http://www.redalyc.org/ pdf/473/47312479003.pdf.

36.- Dickinson S, Hancock D P, Petocz P, Ceriello A, Brand-Miller J. High-glycemic index carbohydrate increases nuclear factor-kappaB activation in mononuclear cells of young, lean healthy subjects. Am J Clin Nutr 2008; 87 (5): 1188-93. DOI: $10.1093 / \mathrm{ajcn} / 87.5 .1188$.

37.- Mehrotra R, Duong U, Jiwakanon S, Kovesdy S P, Moran J, Kopple D J, et al. Serum albumin as a predictor of mortality in peritoneal dialysis: comparisons with Hemodialysis. Am J Kidney Dis 2011; 58 (3): 418-28. doi: 10.1053/j. ajkd.2011.03.018.

38.- Avram M M, Goldwasser P, Erroa M, Fein P A. Predictors of survival in continuous ambulatory peritoneal dialysis patients: The importance of prealbumin and other nutritional and metabolic markers. Am J Kidney Dis 1994; 23: 91-8.

39.- McIntyre C W. Update on peritoneal dialysis solutions. Kidney Int 2007; 71 (6): 486-90. doi:10.1038/sj.ki.5002109.

40.- Huerta A, Ramírez S. Hipoalbuminemia severa: factor de riesgo para peritonitis en pacientes en diálisis peritoneal. Med Int Mex 2010; 26(2): 87-94 http://www.cmim.org/boletin/pdf2010/ MedIntContenido02_03.pdf.

41.- Bucio J, Castañeda T. Gérmenes más frecuentes en peritonitis asociada a diálisis peritoneal en pacientes con insuficiencia renal crónica en el Servicio de Urgencias. Archivo de Medicina de Urgencias 2011; 3 (1): 18-23. http://www. medigraphic.com/pdfs/urgencia/aur-2011/ aur111d.pdf.
42.- Iraj N, Shahrzad O, Mostafa H, Mohammad R, Massih N, Khadijeh M, et al. Epidemiology of culture-negative peritonitis in Iranian patients on Continuous Ambulatory Peritoneal Dialysis. Iranian J Kidney Dis. 2011; 5: 332-7. http:// www.academia.edu/14959121/Epidemiology_ of_culture-negative_peritonitis_in_Iranian_ patients_on_continuous_ambulatory_ peritoneal_dialysis

43.- Predari S C, De Paulis A N, Verón D, Zucchini A, Santoianni J E. Fungal peritonitis in patients on peritoneal dialysis: twenty five years of experience in a teaching hospital in Argentina. Rev Argent Microbiol. 2007; 39: 213-7. https:// www.ncbi.nlm.nih.gov/pubmed/18390154.

44.- Olea T, Hevia C, Bajo M A, Del Peso G, Selgas R. Peritonitis por Pasteurella multocida y Candida albicans. Nefrología. 2006; 26: 136-8. http://www.revistanefrologia.com/ es-publicacion-nef-articulo-peritonitis-porpasteurella-multocida-y-candida-albicans-X0211699506019240

45.- Janakiraman H, Abraham G, Mathew M, Lalitha M K, Bhaskar S. Relapsing peritonitis due to co-infection with Mycobacterium triviale and Candida albicans in a CAPD patient. Perit Dial Int 2007; 27: 311-3. https://www.ncbi.nlm. nih.gov/pubmed/17468481.

46.- Nessim S, Nisenbaum R, Bargman J, Jassal $\mathrm{S}$. Microbiology of peritonitis in peritoneal dialysis patients with multiple episodes. Perit Dial Int 2012; 32 (3): 316-32. doi: 10.3747/ pdi.2011.00058.

47.- García R, García P. Aspectos clínicos y microbiológicos de la peritonitis fúngica en diálisis peritoneal. Nefrología. 2009; 29(6): 506-17. http://www. academia.edu/28245340/Aspectos cl\%C $3 \%$ ADnicos_y_microbiol $\%$ C $3 \%$ B 3 gicos de_la_peritonitis_f $\%$ C $3 \%$ BAngica_en di\%C3\%A1lisis_peritoneal.

48.- Prasad N, Gupta A. Fungal peritonitis in peritoneal dialysis patients. Perit Dial Int. 2005; 25: 207-22. https://www.ncbi.nlm.nih.gov/ pubmed/15981767.

49.- Wiggins K J, Craig J C, Johnson D W, Strippoli G F M. Treatment for peritoneal dialysisassociated peritonitis (Review) Cochrane Database Syst Rev 2008; 23 (1): CD005284. doi: 10.1002/14651858.CD005284.pub3.

50.- Fieren M W. The local inflammatory responses to infection of the peritoneal cavity in humans: their regulation by cytokines, macrophages, and other leukocytes. Mediators Inflamm. 2012; 2012:976241. doi: 10.1155/2012/976241. 